\title{
Improvement of Efficiency and Environmental Impact of a Low-Cost Food Dehydrator
}

\author{
Timothy J. Bowser*, R. Scott Frazier and Raghavendra Rao Kakarala
}

Department of Biosystems and Agricultural Engineering, Oklahoma State University, Stillwater, USA

\begin{abstract}
Energy use and carbon emissions are important factors in food industry and in food dehydration in particular. A low-cost, small-scale dehydrator was tested to determine the impact of ventilation waste heat recovery (VHR) on its energy efficiency and carbon footprint. Cilantro, an important cash crop, was dried under three ventilation conditions while product moisture loss and energy use in the dehydrator were recorded. The ventilation conditions were: without VHR, with VHR, and with VHR and exhaust recirculation. Coefficient of performance (COP) for dehydrator operation under each operating condition was $0.135,0.187$, and 0.194 respectively. Tonnes of carbon dioxide equivalent produced when operating the dehydrator were 20.0,13.1, and 18.0 for the same conditions. COP of the dehydrator increased $39 \%$ when a VHR system was added and carbon emissions equivalent was reduced by $35 \%$. Product drying time was also reduced when the VHR system was added to the dehydrator, increasing the amount of production time available.
\end{abstract}

Keywords: Dehydrator, efficiency, impact, environment, optimize.

\section{INTRODUCTION}

Drying is the process of removing moisture from a product while preserving the quality. Growth of micro organisms can be prevented by removing moisture from foods. The process of moisture removal requires a large amount of energy. For this reason, drying is considered to be one of the most energy intensive unit operations in the agricultural and food processing industries. Use of energy resources has an obvious impact on the environment. Development of alternate fuels, renewable sources and energy efficient technology are important issues related to environmental impact. These reasons explain the need to look into potential energy saving options that can have impact on both economical and environmental issues.

The largest portion of waste heat energy in most convectional dehydration processes appears to be in the exhaust stream. [1]. Waste heat can be recovered and reused to preheat makeup air. One of the common means of heat recovery from the exhaust air stream involves the use of heat exchangers. Heat exchangers are devices used to transfer heat from one stream to another by parallel flow or counter flow between the streams. The principle of a heat exchanger may be observed in everyday equipment like dryers, furnaces, air conditioners, and refrigerators.

In modern homes with air conditioning systems, a lot of energy may be wasted in the form of exhaust air. This energy can be recovered using a ventilation waste heat recovery (VHR) system and used to pre heat the fresh air. VHR equipment is mass produced by several companies for use in newly-constructed homes as a means of energy savings and to improve indoor air quality. VHRs in this classification are known to be economical, durable and reliable. This process

*Address correspondence to this author at the Department of Biosystems and Agricultural Engineering, Oklahoma State University, Stillwater, USA; Tel: 405-744-6688; Fax: 405-744-6313; E-mail: bowser@okstate.edu helps to maintain better indoor temperature conditions during the winter compared to homes without a VHR [2]. A VHR unit designed to recover heat from the exhaust air flow of homes was adapted to recover heat from the exhaust stream of a low cost food dehydrator. The efficiency of the energy recovery from an exhaust stream depends on the change in performance of the dehydrator which is based on the energy utilized for the drying process [1]. The recovered heat from the exhaust stream can be used to pre heat fresh air.

The main objective of this study is to determine and compare performance characteristics of the dehydrator and its carbon emissions with and without sensible heat recovery from the exhaust.

\section{MATERIALS AND METHODS}

A small-scale, low-cost dehydrator with a raw product capacity of about $100 \mathrm{~kg}$ was outfitted with a VHR for this study (Fantech model SHR 2004, Lenexa, Kansas). Construction and operation plans for the dehydrator are available for download at www.fapc.okstate.edu. The dehydrator was designed to be built using off-the-shelf parts by entrepreneurs and small business owners entering the dried meat business. It provides a safe, cleanable, consistent drying environment and the dehydration process has a lethality step included for meat products. The dehydrator also works well for non-meat products like snack foods, fruits, vegetables, seeds, and herbs.

Ventilation of the dehydrator was accomplished by exhausting moist air into the environment through a $15 \mathrm{~cm}$ duct that was fitted with a damper. Fresh air was admitted to the dehydrator though another $15 \mathrm{~cm}$ duct equipped with a backflow preventer. Fig. (1) shows a conceptual drawing of the side view of the dehydrator with ventilation ducts and no heat recovery system. 
The VHR selected for this study has a plastic heatexchange surface that is removable without the use of tools. The heat exchanger is cleaned by immersion in detergent solutions, rinsing and drying. In-line air flow control fans for exhaust and makeup air are included with the VHR.

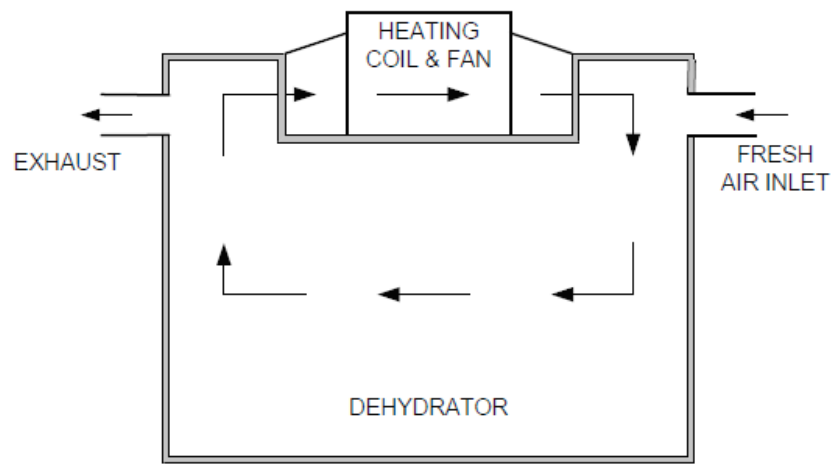

Fig. (1). Conceptual side-view drawing of the dehydrator showing placement of ventilation ducts.

The VHR unit tested is shown in Fig. (2). The VHR was positioned on the roof of the dehydration chamber with ventilation connections made using insulated, flexible, $15 \mathrm{~cm}$ diameter duct. Fig. (3) is a conceptual drawing showing the dehydrator with the installed VHR and air flow direction. All of the heat recovered was sensible heat, as $100 \%$ of the water vapor was exhausted. If the fresh inlet air was cool enough, condensation in the exhaust stream may have occurred, resulting in additional heat recovery. A recycled air flow arrangement was also tested to potentially gain additional heat recovery. Some of the exhaust air was recycled back through the dehydrator and the VHR to allow it time to pick up additional moisture. The recycled air VHR air flow scheme is shown in the conceptual drawing Fig (4). The damper shown in Fig. (4) was manually set to recycle about $70 \%$ of the exhaust air.

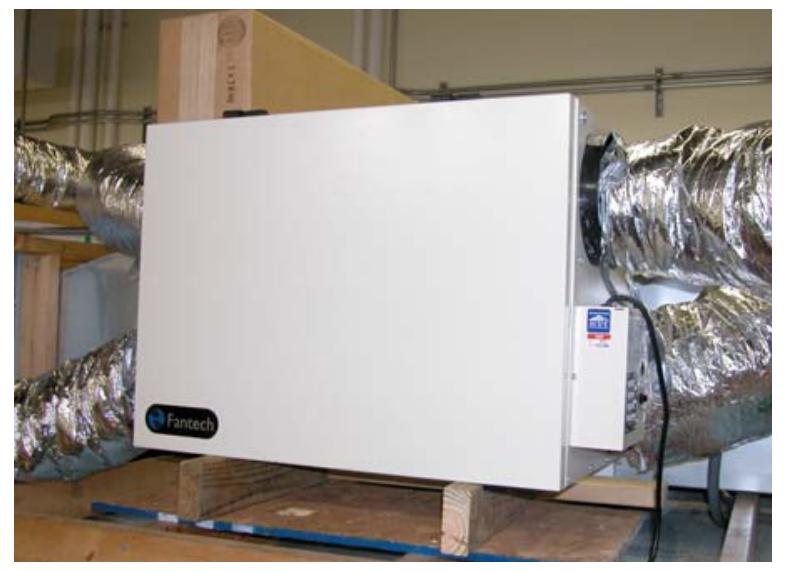

Fig. (2). Ventilation heat recovery (VHR) unit used with the lowcost dehydrator; shown with flexible, insulated duct connections.

Heated water was provided to the dehydrator by a continuous hot-water heater (Takagi Ind. Co., TK-Jr., Irvine, CA) system that was powered by natural gas. Heated water was pumped to a fan coil unit (Triangle Tube, model AHS2125, Blackwood, New Jersey), also mounted on the roof of the dehydrator, where it heated air that was constantly circulated through the dehydrator. After passing through the fan coil unit, the heated water was returned to the hot-water heater in a continuous loop.

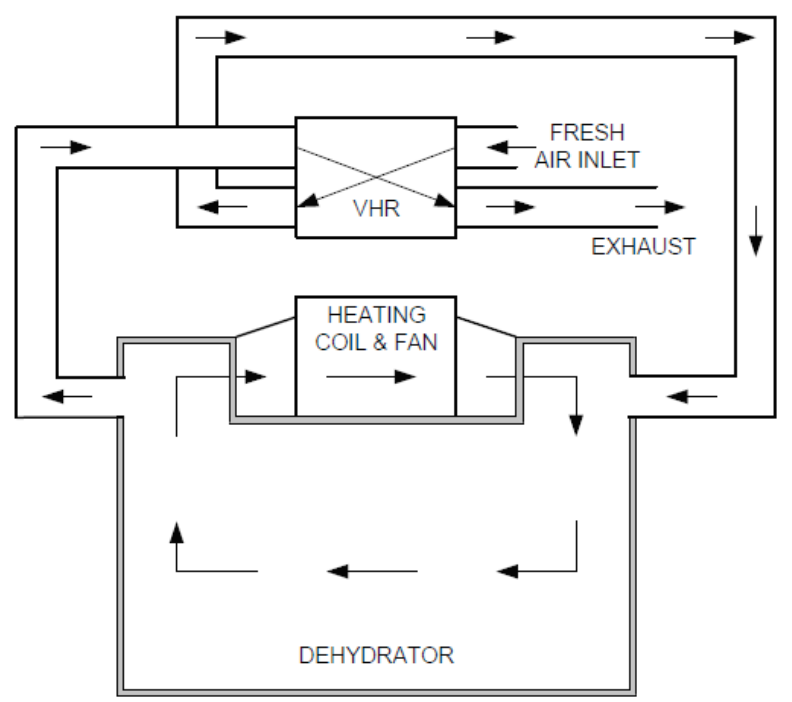

Fig. (3). Conceptual side view of the dehydrator showing air flow and air ducts connecting the VHR unit to the dehydrator.

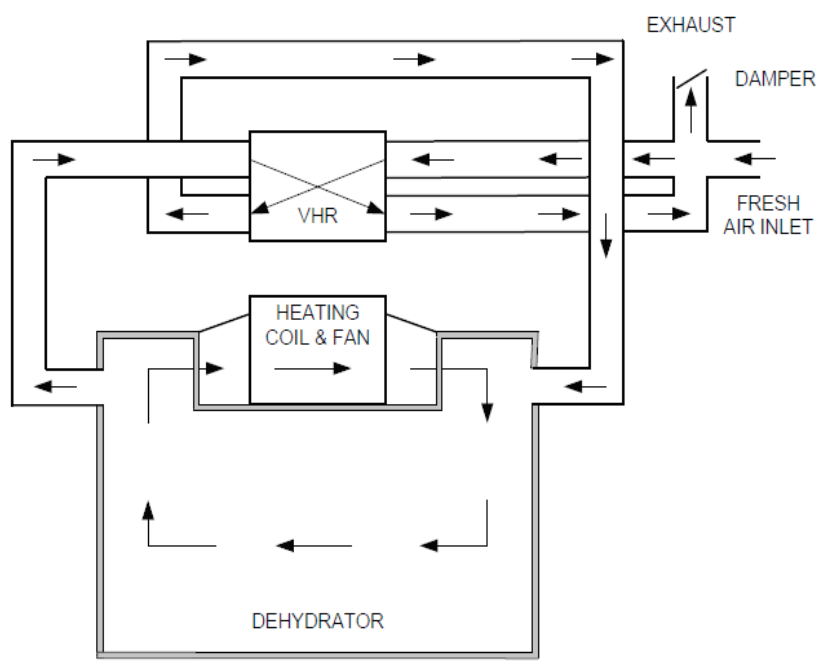

Fig. (4). Conceptual drawing showing a side view of the dehydrator with the recycled air flow scheme.

Resistance temperature detectors (RTDs) (Omega Engineering, Inc., model PT100, Stamford, CT) were used to measure water temperature differential in the continuous loop; one RTD was placed in the pipe after the hot water heater, and the other after the fan coil unit. The RTDs were connected using the method described by [3] to reduce measurement error. Air temperatures were measured using thermocouples (Omega Engineering, Inc., 5TC series, type $\mathrm{K}$, Stamford, CT). All instruments were connected to either a Fluke (Fluke, Hydra 2620A, Everett, WA) or Graphtec (Graphtec GB, Ltd, model GL450, Cheshire, UK) data logger for recording at a frequency of 10 seconds.

Dehydrator setup and operation procedures followed those outlined by Bowser [4]. The dehydrator internal air temperature was set at $75 \mathrm{C}$ for each run. Moisture removal 
was measured directly using a force gauge and live weigh pan system described by Bowser [5].

Cilantro (Coriandrum sativum) was selected as the test product in evaluating the energy efficiency and environmental impact of the dehydrator because of its emerging importance as a cash-crop in Oklahoma, and immediate availability. One live weigh pan and one mobile drying rack (Fig. 5) containing a total of $12 \mathrm{~kg}$ (initial weight) of cilantro was used per drying cycle. The cilantro was fresh-picked from the field by hand cutting the stem with a knife and was water-washed and drip-dried prior to dehydration. Similar procedures for product preparation were used in each experimental drying test.

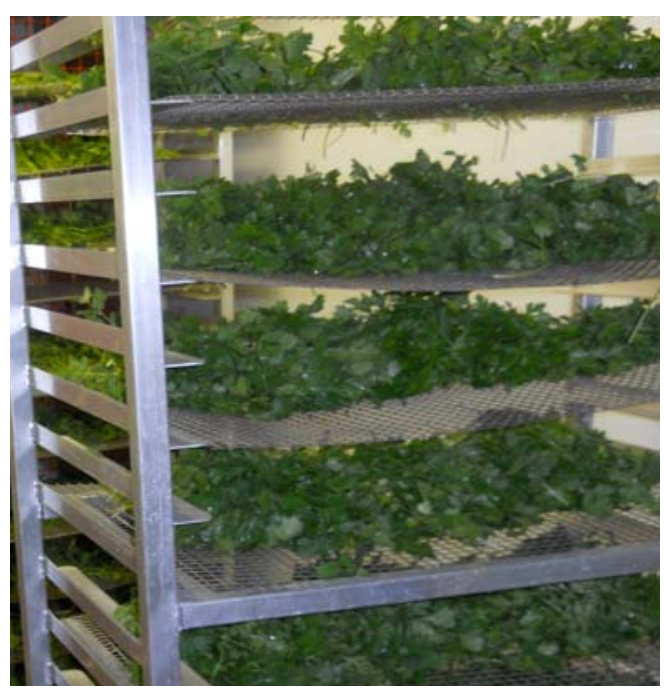

Fig. (5). Mobile drying rack packed with cilantro prior to dehydration. Cilantro was evenly distributed between 10 trays.

Coefficient of performance (COP) is commonly used to determine the efficiency of dehydrators and heat exchangers. COP for the system was calculated during the constant drying rate period for cilantro, and is defined as:

$\mathrm{COP}=$ energy required/energy used

Energy required by the system was calculated as:

Energy required $=$ heat energy input + electrical input.

The major source of heat energy input to the system was through the water heater which was fueled by natural gas (assumed heating value of $38,267 \mathrm{~kJ} / \mathrm{m}^{3}$ [6]) to heat water with an efficiency of $80 \%$. Natural gas consumed was calculated based on the measured amount of heat transferred from the water recirculation system to the dehydrator during the constant drying period for cilantro.

Electrical energy used by the system was measured with an ammeter (Fluke Corp., model 77, Series II, Everett, WA) connected at the main electrical supply to the dehydrator. Electricity was used to operate four fans (two in the dehydrator and two in the VHR) and one water pump used to recirculate hot water in the heating system.

Energy used by the system was needed to evaporate moisture from the product and was taken as $2,385.5 \mathrm{~kJ} / \mathrm{kg}$ of moisture removed. This amount represents the total heat required to evaporate water at $49 \mathrm{C}$, the approximate average surface temperature of the product.
Environmental impact of dehydrator operation was assessed by calculating the carbon footprint of the dehydrator at each operating condition. Calculators were used to estimate the amounts of greenhouse gases produced by stationary combustion sources [7] and purchased electricity [8], based on data collected.

\section{RESULTS}

Drying curves for cilantro at $75^{\circ} \mathrm{C}$ and three different operating conditions are shown in Fig. (6).

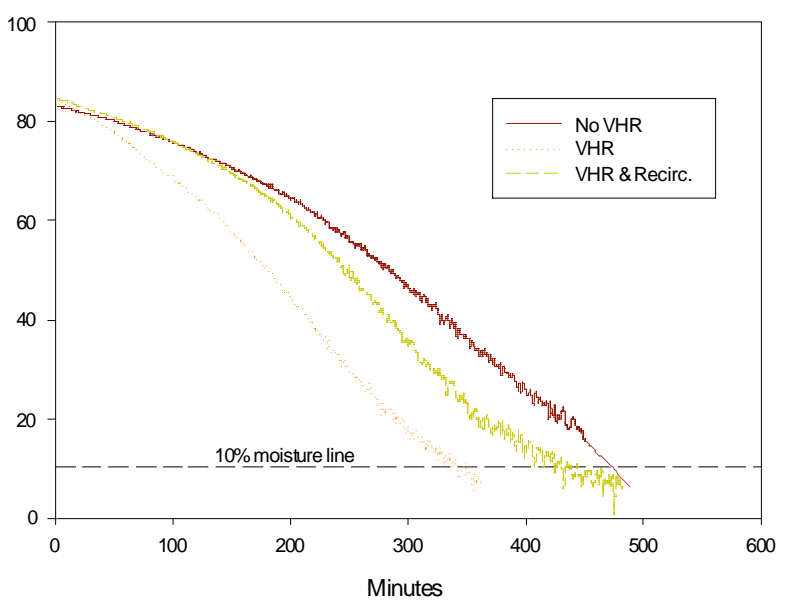

Fig (6). Drying curves of cilantro at $75^{\circ} \mathrm{C}$ and three different operating conditions. $\mathrm{X}$-axis represents the hours of drying and $\mathrm{Y}$-axis represents the percentage moisture content (wet basis).

Coefficient of performance (COP) of the dehydrator and time for the cilantro to reach $10 \%$ moisture content are shown in Table $\mathbf{1}$ for each operating condition of the dehydrator when processing at $75^{\circ} \mathrm{C}$. Only the constant-rate drying period was considered in COP calculations.

Table 1. Coefficient of Performance of the Dehydrator at 75C Drying Temperature and Three Operating Conditions During the Constant Drying Period and Time for the Product to Reach $10 \%$ Moisture Content.

\begin{tabular}{|c|c|c|}
\hline Condition & COP & Time to dry cilantro to 10\% m.c., hr \\
\hline \hline No VHR & 0.135 & 7.86 \\
\hline VHR installed & 0.187 & 5.69 \\
\hline VHR \& recirc. & 0.194 & 7.14 \\
\hline
\end{tabular}

Greenhouse gas emissions from combustion of natural gas and indirect emissions from consumption of purchased electricity were calculated for dehydrating cilantro at $75^{\circ} \mathrm{C}$. Results are shown in Table 2. Emissions were calculated on an annual operating basis, considering a $100 \mathrm{~kg}$ batch of product per day, and 220 production days per year.

\section{DISCUSSION}

Cilantro dried in $28 \%$ less time with the VHR unit installed and functioning without exhaust recirculation compared to the "no VHR" operating condition. This was expected, since the VHR unit forces more fresh makeup air into the dehydrator, lowering the internal humidity. The co- 
Table 2. Estimated Greenhouse Gas Emissions (Annual) from Operation of Dehydrator at $75^{\circ} \mathrm{C}$ to Process Cilantro at Three Operating Conditions

\begin{tabular}{|c|c|c|c|}
\hline \multirow{2}{*}{ Emissions source } & \multicolumn{2}{|c|}{ Emissions at given operating conditions, CO $\mathbf{O}_{2}$ (tonnes) / 100 kg cilantro } \\
\cline { 2 - 4 } & No VHR & VHR installed & VHR with recirculation \\
\hline \hline Purchased electricity & 4.6 & 3.4 & 4.1 \\
\hline Natural gas & 15.4 & 9.7 & 13.9 \\
\hline Combined emissions (total) & 20.0 & 13.1 & 18.0 \\
\hline
\end{tabular}

Table 3. Estimated Value of Inputs Used in Economic Analysis. Annual Savings is Calculated Based on Assumed Operating Schedule and Difference Between Operating Costs of System without and with VHR Unit

\begin{tabular}{|c|c|c|c|}
\hline Input item & Unit & Value, \$ & Annual Savings, \$ \\
\hline \hline Natural gas & $\$ / \mathrm{kW} \cdot \mathrm{hr}$ & 0.030 & 333.43 \\
\hline Electricity & $\$ / \mathrm{kW} \cdot \mathrm{hr}$ & 0.104 & $11,935.00$ \\
\hline Plant operation & $\$ / \mathrm{hr}$ & 25 & 173.41 \\
\hline Carbon emissions & $\$ /$ ton CO $\mathrm{C}_{2}$ & 25 & NA \\
\hline Installed cost of VHR system & $\$$ & 7 & NA \\
\hline Discount rate & $\%$ & 5 & NA \\
\hline Period & year & 100 & NA \\
\hline Cilantro & Kg/batch & & \\
\hline
\end{tabular}

efficient of performance (COP) of the dehydrator increased, providing a $39 \%$ improvement in the COP compared to the dehydrator operating without the VHR. The difference between the COP values for the dehydrator operating with the VHR installed with and without exhaust gas recirculation was less than $5 \%$, indicating that exhaust gas recirculation did not make a significant difference.

Operation of the dehydrator with the VHR (without exhaust gas recirculation) reduced annual estimated carbon emissions $\left(\mathrm{CO}_{2}\right.$ equivalent) of the system by $35 \%$ compared to the "no VHR" operating condition. Carbon emissions associated with natural gas use were reduced by $37 \%$, while emission associated with purchased electricity were reduced by $28 \%$.

Recovery of ventilation waste heat in a dehydration process for cilantro at $75^{\circ} \mathrm{C}$ significantly reduced process time, increased energy efficiency and reduced environmental impact. A financial analysis of the installed HRV unit (without exhaust recirculation) was estimated by assuming costs given in Table $\mathbf{3}$ and calculating a simple payback and net present value for the cash flow. Utility costs in Table $\mathbf{3}$ were taken as current average values for commercial utility customers in the U.S. for electricity [9] and natural gas [10]. Carbon savings (based on reduction of energy use after installing an HRV) are included at an assumed value. It has also been assumed that the performance of the dehydrator with a $100 \mathrm{~kg}$ batch of cilantro would be identical to the performance of the $12 \mathrm{~kg}$ test batch. We believe that the final assumption given is reasonable based on our experience operating the low-cost food dehydrator with a wide range of product batch sizes.
Simple payback for the investment requires about 124 hours of dehydrator operation time ( 22 batches of cilantro at $100 \mathrm{~kg} / \mathrm{batch}$ ). Net present value for the VHR installation on a dehydrator with an annual operating basis of 1 batch of product per day and 220 production days per year is $\$ 52,232$ (assuming no residual value for the VHR unit and no maintenance costs). In the previous evaluation, reduction of processing time played a major role in the economic analysis; this may not be the case for many dehydrators. If processing time was equal for dehydrator operation with and without the VHR, then the simple payback due to energy and carbon emissions savings would be 1.29 years with a net present value of $\$ 4,755.27$. Depending on the value of production time, energy costs, and carbon emissions, a ventilation waste heat recovery unit for a dehydrator may be a profitable investment.

\section{ACKNOWLEDGEMENTS}

The authors acknowledge the following groups at the Oklahoma State University, Stillwater, for their important contributions to this work: The Robert M. Kerr Food \& Agricultural Products Center for providing the facility and funding for the study; and, the Department of Biosystems \& Agricultural Engineering for providing mechanical and electrical services in support of the experimental setup. The World Resources Institute is also acknowledged for making available free copies of their valuable GHG Protocol tools.

\section{REFERENCES}

[1] Krokida MK, Bisharat GI. Heat Recovery from Dryer Exhaust Air. Dry Tech 2004; 22(7): 1661-74. 
[2] Zhang Y, Jiang Y, Zhang LZ, Deng Y, Jin Z. Analysis of thermal performance and energy savings of membrane based heat recovery ventilator. Energy 2000; 25: 515-27.

[3] Wynne J. RTDs provide differential temperature measurement. EDN magazine. Internet: http://www.edn.com/file/19731122100di.pdf (accessed: May 17, 2011).

[4] Bowser TJ. Construction and operation manual for: Low-cost, safe dehydrator for small and very small meat processors. 2007. Oklahoma State University, Food and Agricultural Products Center. Internet: http://fapc.okstate.edu/files/DehydratorManualV1.pdf (accessed: April 18, 2008)

[5] Bowser TJ, Weckler PR. Validation guidelines for a jerky pasteurization process in a low-cost dehydrator. Open Food Sci J 2008; (2): 43-8.
[6] International Energy Agency. 2010. Key world energy statistics. Paris, France: pp. 60.

[7] World Resources Institute. 2009. GHG Protocol tool for stationary combustion. Version 4.0. Available for download at: http://www.ghgprotocol.org/calculation-tools/all-tools.

[8] World Resources Institute. 2009. GHG Protocol tool for purchased electricity. Version 4.0. Available for download at: http://www.ghgprotocol.org/calculation-tools/all-tools.

[9] U.S. Department of Energy. 2011. Energy explained. Internet: http://www.eia.doe.gov/energyexplained/index.cfm?page=electricit y_factors_affecting_prices. (Accessed: 5, May 11).

[10] U.S. Department of Energy. 2011. Energy explained. Internet: http://www.eia.doe.gov/dnav/ng/ng_pri_sum_dcu_nus_m.htm.

(Accessed: 5, May 11).

(C) Bowser et al.; Licensee Bentham Open .

This is an open access article licensed under the terms of the Creative Commons Attribution Non-Commercial License (http://creativecommons.org/licenses/by-nc/3.0/) which permits unrestricted, non-commercial use, distribution and reproduction in any medium, provided the work is properly cited. 